\title{
Estimates of Metal Deformability
}

\author{
Albina Zharmukhambetova ${ }^{1, *}$ and Svetlana Barannikova ${ }^{1,2}$ \\ ${ }^{1}$ Institute of Strength Physics and Materials Science SB RAS, 634055, Akademicheskii pr., 2/4, Tomsk, \\ the Russian Federation \\ ${ }^{2}$ Tomsk State University of Architecture and Building, Tomsk, the Russian Federation
}

\begin{abstract}
This paper is devoted to the study of the laws of the deformation behavior of metals, determined by carrying out standardized uniaxial tensile tests. The distribution of various strength grade metals by strain resistance was analyzed. It was taken into account that in the thermodynamic aspect the deformation process is a dissipative effect. In that regard, the magnitude of deformation work, determined by the area of the tension diagram, serves as a basis for assessing the deformability criteria of metals. It was found, that the normalized specific work of deformation linearly increases with strength. As well as it was established that localization of plastic flow is related directly to the characteristics of the tension diagram under solid deformation. In the applied aspect, the numerical values of the compliance criterion can be used to predict the materials behavior of different strength grade during mechanical processing (metal forming and cutting) or operation.
\end{abstract}

\section{Introduction}

Computational evaluation methods for the stress-strain state of materials and products are often complex in dealing with problems of stress and strain distribution in samples or real products of complex shape especially when the material has an internal structure. This structure can change during the deformation due to successive processes of formation and relaxation of stress concentrators. As a result of mechanical tests, stress-strain curves are obtained, which qualitatively and quantitatively characterize the material's response to loading, and by which determine the strength and ductility of materials.

The existing approaches of plasticity problem differ in the structure of the models used [1-5], which most often than not have a pragmatic meaning and are designed to provide a solution of specific problems associated with plastic deformation. In most cases, this approach does not promote understanding of the nature of plasticity phenomenon in general. The plastic flow often begins as a spatially localized process, as exemplified by the deformation on the yield plateau [4,5], which is localized in the Luders band. The deformation process usually also ends in the localized form - the nucleation of a macroscopic neck. It is likely that at the intermediate stages, the deformation also proceeds in the form of a localization, and inadequate data is caused by the features of the experimental techniques used for the study. At deformation localization, an initially homogeneous medium

\footnotetext{
* Corresponding author: zharmukhambetova@gmail.com
} 
is spontaneously divided into deformable and non-deformable volumes, the boundaries of which can be movable [6]. It is obvious that in localization zones and between them, plastic deformation develops in different ways. However, traditional physics of material plasticity, which is essentially based on the dislocation theory [9], uses electron microscopic analysis in these cases, oriented to the study of small volume of a deformable crystal $\sim 10^{-17} \mathrm{~m}^{3}$. In recent years, many authors experimentally observed patterns of localized plastic flow during deformation. In [1-8], laser interferometry, digital image correlation method, and thermal imaging systems were used for this.

Information on the strength properties of materials may be of interest to two consumer groups. Firstly, engineers need it to select a material based on its physical and mechanical properties. Secondly, researchers also can't do without quantitative characteristics of stressstrain dependency to find explanations of the nature of materials properties. This explains the miscellaneous interest in the valuation problem of the mechanical characteristics by the load curves and requires the unification of processing methods of plastic flow curves.

Thus, this work is devoted to the study of the laws of the deformation behavior of metals, determined in standard uniaxial tension tests, as well as to search for evidence of the existence of plastic flow localization from yield strength to fracture. The basis for evaluation of the deformability of metals criteria will be deformation work, determined by the area of the tension diagram.

\section{Experimental part}

Mechanical uniaxial tension tests were carried out in a universal test machine (Instron-1185) at a constant speed of moving gripping $\mathrm{Vm}=0.2 \mathrm{~mm} / \mathrm{min}$ on a wide range of metals and alloys $(\mathrm{Cu} ; \gamma$-Fe; $\mathrm{Ni} ; \mathrm{Sn} ; \mathrm{Nb} ; \mathrm{Zn} ; \mathrm{Mg} ; \mathrm{V} ; \alpha$-Fe; $\mathrm{Zr} ; \mathrm{Al}$; Ti; Mo) with various characteristics of durability and plasticity [9]. The load curves of the studied materials are presented in Figure 1. In the thermodynamic representation the macroscopic result of metal deformation (it's a change in shape and size) is a dissipative process, that is the transition of a part of the kinetic energy to the internal energy of the deformable metal, which is realized in the formation of a certain internal structure [10-12]. In so doing, the amount of energy absorbed during deformation is determined by the work of deformation, and absorption rate defines the resistance of the metal to deformation [13]. In the case of tension test, the deformation work is numerically equal to the surface area under stress-strain curve, and is the sum of the work of elastic and plastic deformation.

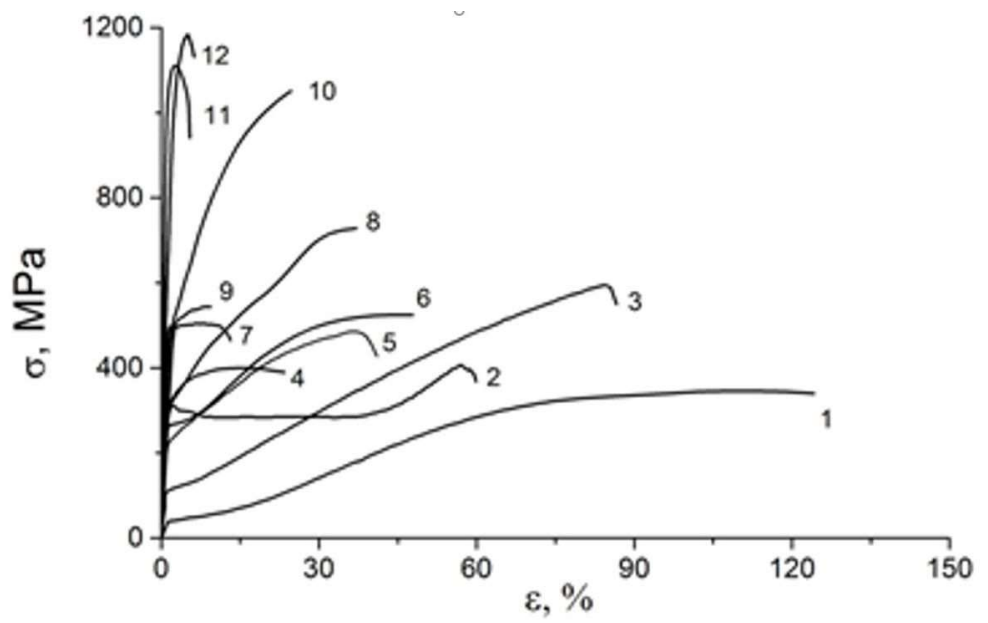


Fig. 1. Deformation curves of the test materials: $1-\mathrm{Cu} ; 2-\gamma$-Fe; $3-\mathrm{Ni} ; 4-\mathrm{Sn} ; 5-\mathrm{Nb} ; 6-\mathrm{Zn} ; 7-$ $\mathrm{Mg} ; 8-\mathrm{V} ; 9-\alpha-\mathrm{Fe} ; 10-\mathrm{Zr} ; 11-\mathrm{Al} ; 12-\mathrm{Ti} ; 13-\mathrm{Mo}$.

Experimental evidence of the localized plastic flow was obtained using a double-exposure speckle photography method specially adapted for the study of deformation in photographic or digital versions [6-8]. This technique enables to reconstruct the field of displacement vectors $r(x, y)$, that occurs when the strain increases $\delta \varepsilon \approx 10^{-3}$ on the sample surface, and to calculate all components of the plastic distortion tensor:

$$
\beta_{i j}=\nabla \boldsymbol{r}(x, y)=\varepsilon_{i, j}+\omega_{z}=\left\|\begin{array}{ll}
\varepsilon_{x x} & \varepsilon_{x y} \\
\varepsilon_{y x} & \varepsilon_{y y}
\end{array}\right\|+\omega_{z} .
$$

The results calculated by standard procedures are presented in the dependences of the components of the plastic distortion tensor $\varepsilon_{x x}, \varepsilon_{y y}, \varepsilon_{x y}=\varepsilon_{y x}$ and $\omega_{z}$ on the $\mathrm{x}$, y coordinates and time $t$, which are calculated from the initial data using specially developed programs. This procedure visualizes the localization patterns in a plastic flow (Fig. 2a). A quantitative analysis of patterns is possible using X-t diagrams (dependence of the coordinates of the deformation fronts $\mathrm{X}$ on time $\mathrm{t}$ ), by which the characteristic spatial $\lambda$ and time $\mathrm{T}$ periods of the process can be measured, as well as the speed of the deformation front $V_{a w}=\lambda / T$ can be estimated (Fig. 2b).

The velocity of the zones of localized plastic flow is determined by the strain hardening coefficient of the material [6-8]

$$
V_{a w}=V_{0}+\Xi / \theta \sim 1 / \theta
$$

where $V_{0}$ and $\Xi$ are constants. The ratio (2), which is valid for the stage of linear strain hardening, covers all the test materials, the deformation curve of which $\sigma(\varepsilon)$ contains this hardening stage. In all cases the observed values of the velocities of the zones of localized plastic flow and the spatial period are $10^{-5} \leq V_{a w} \leq 10^{-4} \mathrm{~m} / \mathrm{s}$ and $\lambda \sim 5 \ldots 10 \mathrm{~mm}$ respectively.
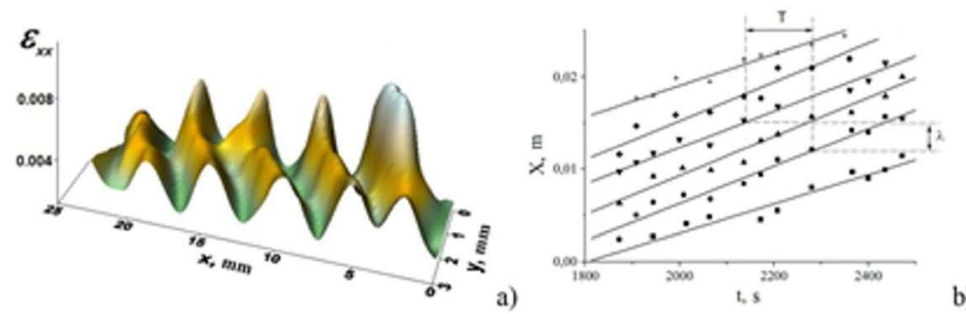

Fig. 2. The example of pattern of localization of plastic low.

The product of $\lambda V_{a w}$ (complex parameter of plasticity) characterizes the process of plastic flow [6-8] and has dimension of kinematic viscosity $\mathrm{m}^{2} / \mathrm{s}$.

In this work the wide range of test metals is justified by the need to search for reliable links between the plasticity parameter $\lambda V_{a w}$ and mechanical characteristics.

\section{Results and discussions}

The stress-strain curves (Figure 1) obtained as a result of mechanical tests were fitted using software package Table Curve in the form of fourth-order polynomials:

$$
\sigma_{i}=a_{0}+a_{1} \cdot \Delta \varepsilon_{i}+a_{2} \cdot \Delta \varepsilon_{i}^{2}+a_{3} \cdot \Delta \varepsilon_{i}^{3}+a_{4} \cdot \Delta \varepsilon_{i}^{4}
$$


At least 15 points were used to do so. The polynomial was used as an integrand to calculate the surface area under stress-strain curve, that is, the deformation work. An inversely proportional relationship between the deformation work on an uniform field of plastic deformation and the ratio of the yield limit to the ultimate strength of test materials was established with a correlation coefficient of 0.83 (Figure 3).

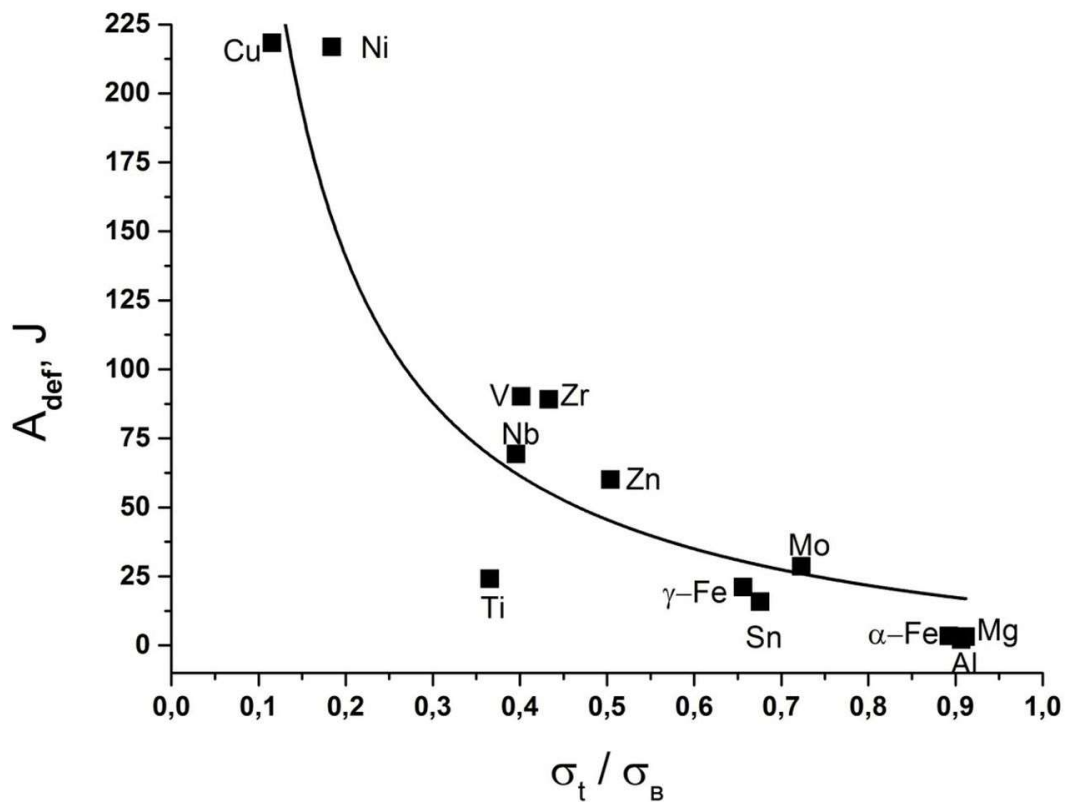

Fig. 3. The dependence of the deformation work on the ratio of yield limit $\sigma_{t}$ to ultimate strength $\sigma_{B}$

To estimate the mechanical deformability of metals, the ratio of deformation work of uniform plastic deformation (from yield limit to ultimate strength of materials) to the deformable material volume calculated as the product of the initial values of the area and working length of each sample was determined. Thus, the specific work values of uniform plastic deformation were determined as:

$$
a_{\text {unif }}=A_{\text {unif }} / V_{\text {def }}
$$

The specific work reflects the material deformability in terms of the limiting work that is irreversible for shaping of the given metal in the individual stages [9], as such it quantitatively reflects the energy dissipation. Another reflection of the material deformability may be the "compliance" proposed in [14] as a resistance to deformation as the deformation due to external factors increases. This quality of metal is defined as the ratio of the specific work to the deformation corresponding to the ultimate strength:

$$
k_{\text {unif }}=\alpha_{\text {unif }} / \varepsilon_{B}
$$

It is actually characterized by the rate of energy dissipation under uniaxial tension.

A linear relationship between the normalized specific work $\mathrm{k}_{\text {unif }}$ and the ultimate strength of the studied metals $\sigma_{B}$ was established with a high correlation coefficient of 0.9 (Figure 4). The quantity of specific energy $\mathrm{mJ} / \mathrm{mm}^{3}$ required to increase the deformation under tension by $1 \%$ is taken as the conventional units of the coefficient $\mathrm{k}_{\text {unif }}$. 


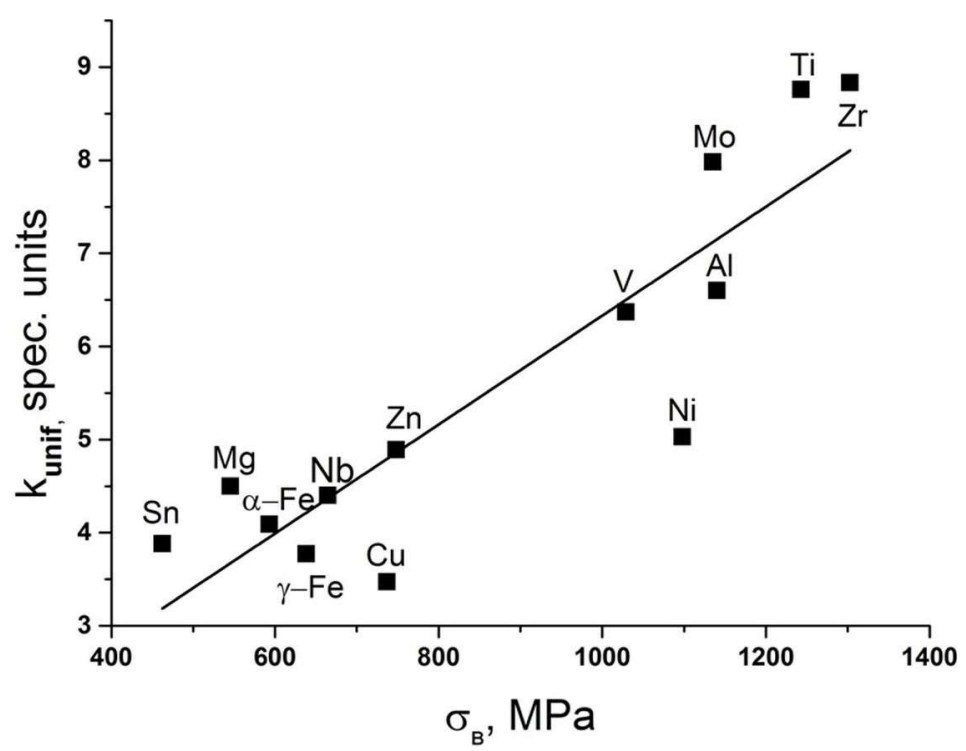

Fig. 4. The dependence of the coefficient kunif on the ultimate strength

The table below shows the experimentally obtained values, as well as the calculated specific work and its normalized value for all studied metals.

The amount of stored energy can be also calculated from the stress-strain curves. A geometric analysis of the stress-strain curve allows one to determine a part of the energy associated with inhomogeneous plastic deformation as shown in [15]. The figure 5 shows a graph of the calculated values depending on the true strain for a number of studied metals. The solid line represents the approximations in the form of second-order polynomials $\varepsilon_{s g i}=a_{0}+a_{1} \cdot \Delta \varepsilon_{i}+a_{2} \cdot \Delta \varepsilon_{i}{ }^{2}$, using at least 8 points for this.

Table 1.

\begin{tabular}{|c|c|c|c|c|c|c|c|}
\hline \multirow[t]{2}{*}{ metal } & $\sigma_{0.2}$ & $\sigma_{\mathrm{B}}$ & \multirow[t]{2}{*}{$\varepsilon_{\mathrm{B}}$} & \multirow[t]{2}{*}{$\varepsilon_{\mathrm{K}}$} & $\mathrm{A}_{\text {def }}$ & $\mathrm{A}_{\text {unif }}$ & $\mathrm{k}_{\text {unif }}$ \\
\hline & \multicolumn{2}{|c|}{$\mathrm{MPa}$} & & & $\mathrm{J}$ & $\mathrm{mJ} / \mathrm{mm}^{3}$ & spec. units \\
\hline $\mathrm{Mg}$ & 471.4 & 545.4 & 0.078 & 0.124 & 3.10 & 35.3 & 4.5 \\
\hline $\mathrm{Al}$ & 1021 & 1140 & 0.025 & 0.053 & 2.01 & 16.8 & 6.6 \\
\hline $\mathrm{Ti}$ & 431.1 & 1243 & 0.049 & 0.060 & 24.10 & 43.1 & 8.76 \\
\hline $\mathrm{V}$ & 332.4 & 1029 & 0.309 & 0.315 & 90.07 & 197.2 & 6.37 \\
\hline$\alpha-\mathrm{Fe}$ & 493.4 & 593 & 0.087 & & 3.39 & 35.4 & 4.09 \\
\hline$\gamma-\mathrm{Fe}$ & 269.9 & 638.5 & 0.451 & 0.469 & 21.02 & 170.3 & 3.77 \\
\hline $\mathrm{Ni}$ & 110.9 & 1098 & 0.612 & 0.624 & 216.68 & 308.1 & 5.03 \\
\hline $\mathrm{Cu}$ & 40.5 & 737 & 0.757 & 0.807 & 218.15 & 262.8 & 3.47 \\
\hline $\mathrm{Zn}$ & 268.1 & 748.1 & 0.341 & 0.391 & 59.94 & 166.7 & 4.89 \\
\hline $\mathrm{Zr}$ & 465.2 & 1303 & 0.218 & & 89.02 & 192.4 & 8.83 \\
\hline $\mathrm{Nb}$ & 161.2 & 664.5 & 0.315 & 0.344 & 69.31 & 138.5 & 4.4 \\
\hline Mo & 678.6 & 1135 & 0.216 & & 28.59 & 172.6 & 7.98 \\
\hline $\mathrm{Sn}$ & 271.8 & 462.2 & 0.145 & 0.210 & 15.81 & 56.4 & 3.88 \\
\hline
\end{tabular}




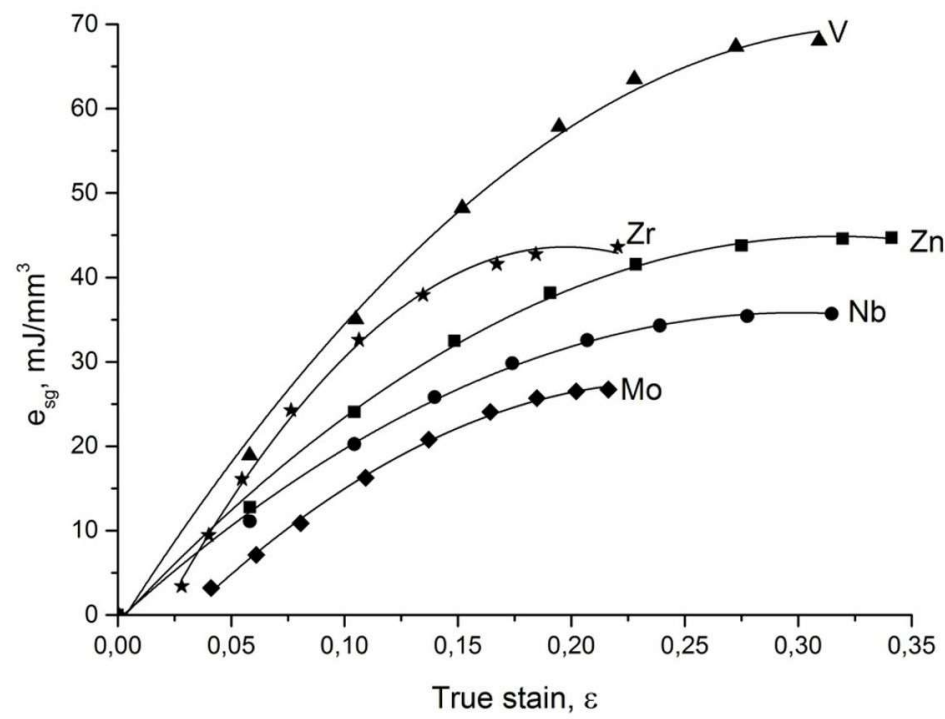

Fig. 5. The dependence of the accumulated energy, calculated from the stress-strain curve, on the true strains.

Based on the results obtained by the speckle-photography method, it can be argued that the localized plastic flow of solids is realized as a sequence of strain localization patterns in the form of local strain distributions, which in turn evolves along the plastic flow curve $\sigma(\varepsilon)$ of metals and alloys. That is, the staging of the deformation process alone can be caused by a change in the autowave deformation mechanisms [6-8]. it was established that the parameter $\lambda V_{a w}$, which characterizes the plastic properties of the studied metals, correlates with the values of the elastic constants and energy characteristics. The result of the analysis of the behavior of parameter $\lambda \mathrm{V}_{\text {aw }}$ identified that this value is inversely proportional to the bulk modulus $\mathrm{K}$, whose values were borrowed from reference books [10], and the deformation work $A_{\text {def }}$ (Fig. 6).
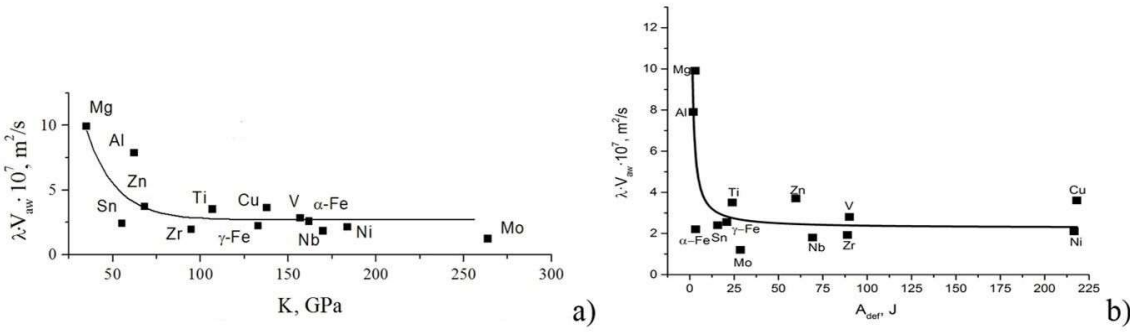

Fig.6. The dependence of the plastic parameters on the compression modulus (a) and the deformation work under (b) uniaxial loading.

\section{Conclusions}

As a result of the research conducted, an analysis of the material distribution of various strength classes by strain resistance was carried out. At the same time, the yield limit is 40 $1050 \mathrm{MPa}$, and the ultimate strength is $460-1240 \mathrm{MPa}$ for all studied materials used for construction products. It was found that during deformation of a solid, the parameters of 
localization of plastic flow are related to the characteristics of the load curves of metals, as follows: the parameter $\lambda V_{\mathrm{aw}}$ is inversely proportional to the bulk modulus and the deformation work. As well as it has been established that the specific work of concentrated deformation normalized to the corresponding deformation, $\mathrm{k}_{\text {unif, }}$ increases linearly with increasing strength. In the applied aspect, the numerical values of $\mathrm{k}_{\text {unif }}$ can be used to predict the materials distribution of different strength according to the behavior in working and mechanical operation (metal forming and cutting). Metal, in which deformation is accompanied by increased specific work, are more serviceable in operation and technological in pressure treatment, but less technological in cutting. At the same time, metals characterized by high values of compliance criteria are also more efficient in operation, but less technological in both pressure treatment and cutting. Moreover, the predicted conditions of the forming operation should provide a mechanical effect on the metal that does not result in the deformation localization, whereas the predicted conditions of the cutting require destruction (with a prelocalization). Therefore, in the case of pressure treatment, the coefficient of $\mathrm{k}_{\text {unif }}$ is a characteristic of manufacturability. Taking into account the wide range of materials strength, the criterion of deformability in figure 3 can be considered quite universal criterion for ferrous and non-ferrous metals and the magnitude of $k_{\text {unif }}$ can be estimated from known quantity of the ultimate strength.

Acknowledgements. The work was carried out within the framework of the Program of Fundamental Scientific Research of the State Academies of Sciences for 2013-2020.

\section{References}

1. T.V. Tretyakova, V.E. Wildemann, Fract. Struct. Integrity, 11:42, 303 (2017)

2. A.A. Shibkov, M.F. Gasanov, M.A. Zheltov, A.E. Zolotov, V.I. Ivolgin, Int. J. Plasticity, 86, 37 (2016)

3. A. Kostina, Y. Bayandin, O. Naimark, O. Plekhov, Key Eng. Mater. 592:593, 205 (2014)

4. B. Reyne, P.-Y. Manach, N. Moes, Mater. Sci. Eng. A 746, 187 (2019)

5. N.P. Kobelev, M.A. Lebyodkin, T.A. Lebedkina, Metall. Mater. Tran., 48:3, 965 (2017)

6. L.B. Zuev, S.A. Barannikova, A.G. Lunev, Prog. Phys. Met. 19:4, 379 (2018)

7. L.B. Zuev, S.A. Barannikova, A.G. Lunev, S.V. Kolosov, A.M. Zharmukhambetova, Russ. Phys. J. 61, 1709 (2019)

8. L.B. Zuev, S .A. Barannikova, Solid State Phenom. 172:174, 1279 (2011)

9. R.E. Newnham Properties of Materials (Oxford: University Press, 2005)

10. A.A. Kostina, O.A. Plekhov, Solid State Phenom. 243, 43 (2016)

11. E.G. Pashinskaya, A.V. Zavdoveev, L.S. Metlov, Y.I. Nepochatikh, A.A. Maksakova, V.M. Tkachenko, Mater. Phys. Mech. 24:2,163 (2015)

12. Yu.V. Bugrov, Ind. Lab. Diagn. Mater. 3, 66 (2012)

13. R.E. Gliner, V.N. Dubinskii, E.B. Katyukhin, V.A. Pryanichnikov, Steel Transl. 47:9, 589 (2017)

14. G.A. Smirnov-Alyaev Resistance of materials to plastic deformation (Leningrad: Machine industry, 1978)

15. W. Oliferuk, M. Maj, Eur. J. Mech. - A/Solids. 28:2, 266 (2009) 